\title{
Structure preservation in comparatives*
}

\author{
Alexis Wellwood \\ University of Southern California
}

\begin{abstract}
Comparatives can invoke various dimensions for comparison, but not anything goes: more coffee invokes volume or weight, but not temperature, while more coffees invokes number, but not volume or weight. In general, the extant literature assumes that the difference between more coffeelcoffees reflects a morphosyntactic ambiguity of more, such that it spells out MUCH-ER with bare nouns, and MANY-ER with plural nouns. Semantically, MUCH introduces a variable over measure functions, with constraints, whereas MANY introduces a cardinality function. I argue for an alternative, univocal theory based on the decomposition MUCH-ER, and account for the observed patterns of constrained variability by means of a stronger condition on the selection of measure functions than has previously been proposed.
\end{abstract}

Keywords: comparatives, monotonicity, measurement, plurality, number, decomposition

\section{Introduction}

In a degree semantics framework, ${ }^{1}$ it is uncontroversial to assume that part of the meaning of more as it occurs in nominal comparatives like (1) introduces a measure function, whereas this role is played by the gradable adjective hot in adjectival comparatives like (2). This paper is concerned with the identity and meaning of the part of more that does this.

(1) a. Ann bought more coffee than Bill did.

b. Ann bought more coffees than Bill did.

a. Ann's coffee is hotter than Bill's is.

b. Ann bought hotter coffees than Bill did.

* I would like to thank Jeremy Goodman, Michael Glanzberg, Barry Schein, Una Stojnić, and Paolo Santorio for helpful recent discussion.

1 I do not motivate this framework here (for early works see Seuren 1973, 1984; Cresswell 1976), and I assume the variant usually attributed to Kennedy 1999 in which, e.g., tall denotes a 'measure function' of type $\langle e, d\rangle$. Since any possible scopal interactions with the -er morpheme will not be at issue, I won't appeal to the higher-typed variant usually attributed to Heim 1985, 2001 in which tall denotes a 'degree relation' of type $\langle d,\langle e, t\rangle\rangle$, itself embedding a measure function. 
Structure preservation

I focus this investigation on two generalizations. The first, Monotonicity, captures the fact that while the dimension for comparison with more can vary within predicates $P$, as well as across predicates-e.g., (1a) can express a comparison by volume or weight, while (1b) expresses a comparison by number - the only permissible dimensions are ones that preserve strict ordering relations on the measured domain $D_{P}$-e.g., (1a) cannot be understood as a comparison by temperature, since this might involve mapping a portion of coffee and its subparts to the same degree.

Monotonicity

Schwarzschild 2002, 2006

Comparatives show variable dimensionality, but permissible dimen-

sions for more $P$ preserve strict ordering relations on $D_{P}$.

The second generalization, Number, captures the fact that the only permissible dimension for comparison when more occurs with plural nouns is number, or cardinality. ${ }^{2}$ This is true for (1b) with coffees, despite the availability of the volume and weight dimensions when its head noun coffee occurs without the plural morpheme. It is also true for nouns like ideas, where the corresponding bare form makes no dimensions available, or at least not easily (cp. ?more idea and more ideas). Importantly, the restriction to number with more $P-s$ also adheres to Monotonicity.

\section{Number Hackl 2001; Bale \& Barner 2009 \\ Plural N comparatives permit only number-based comparisons.}

The literature on nominal comparatives tends to assume that these patterns arise due to an ambiguity of more. In brief, an Ambiguity account (found in various forms in Bresnan 1973; Cresswell 1976; von Stechow 1984; Heim 1985; Rett 2008; Hackl 2001; Bhatt \& Pancheva 2004; Solt 2015, among others) holds that more is homophonous between the morphosyntactic complexes MUCH-ER and MANY-ER which selectively occur with bare and plural nouns, respectively. On such a view, the semantics of MUCH will be freer than MANY in terms of dimensionality, but that semantics will be constrained so as to capture the Monotonicity generalization.

I argue for a different, univocal account, building on Schwarzschild's (2002; 2006) theory of dimensional restrictions for sentences like (1a). My goal in extending that theory is to ensure that sentences like (1b) are restricted to comparison by cardinality. In other words, where Schwarzschild's theory permits cardinality for more coffees, I aim to enforce it. On such a Univocality account, the Number generalization is viewed as a subcase of Monotonicity, and to capture it, we need

2 There is a well-known class of apparent counter-examples, the so-called 'mass plurals'. See, among others, Acquaviva 2008; Schwarzschild 2012; Solt 2015; and Wellwood 2014. 


\begin{tabular}{l|lll}
\hline account & string & morphosyntax & semantics \\
\hline \hline Ambiguity & more $N$ & $\mathrm{MUCH}_{\mu}$-ER N & $\ldots \sigma(\mu)(x) \ldots$ \\
& more $N s$ & MANY-ER N-PL & $\ldots$ cardinality $(x x) \ldots$ \\
\hline Univocality & more $N$ & $\mathrm{MUCH}_{\mu}$-ER N & $\ldots \sigma(\mu)(x) \ldots$ \\
& more $N s$ & $\mathrm{MUCH}_{\mu}$-ER N-PL & $\ldots \sigma(\mu)(x x) \ldots$
\end{tabular}

Table 1 Aligning strings, structures, and semantics on the two accounts.

only augment Schwarzschild's condition on the selection of measure functions with an additional condition that further restricts that selection.

The two accounts can be assumed to overlap in how they analyze $\mathrm{MUCH}$, such that it carries an index $\mu$ of the measure function type. The value of this variable in (i.e., $\sigma(\mu)$, for an assignment $\sigma$ ) in sentences like (1a) is constrained by Schwarzschild's condition. The accounts part ways for sentences like (1b). Where the Univocality account further constrains the selection of $\sigma(\mu)$, the Ambiguity account posits a distinct lexical primitive, MANY, to itself introduce a specific measure function. The similarities and differences between these accounts are summarized in Table 1. ${ }^{3}$

Before turning to my argument, it is important to note that the range of data that these accounts apply to extends beyond comparative constructions with more, and beyond nominal comparatives to include (at least) verbal comparatives. ${ }^{4}$ That is, both Monotonicity and Number appear to capture relevant facts about the interpretation of sentences in which the expression introducing the comparative relation is as, too, enough, etc.; see (3) for examples. The same can be observed for verbal comparatives; see (4).

(3) Nominal; Monotonicity and Number
a. as much coffee
b. too many coffees
volume, weight, *temperature
cardinality, *volume, ${ }^{*}$ weight

(4) Verbal; Monotonicity and Number
a. run on the track as much
b. run to the track more/as many times
distance, duration, *speed cardinality, *dist., *dur.

In what follows, I argue that the Univocality account provides a better explanation for Monotonicity and Number, as well as satellite facts. The last two sections of

3 By $x x, y y, \& c$. in Table 1 and below, I intend only visual clarity of talk of pluralities - the nature and formal representation of plurality won't be at issue here. See Liebesman 2016, though, for recent discussion and citations of this notation, drawn from plural logic.

4 Dunbar \& Wellwood 2016 and Cariani, Santorio \& Wellwood 2018; submitted further suggest extensions to adjectival comparatives. See also Fults 2006. 
Structure preservation

the paper discuss further considerations that might recommend it: (i) a univocal theory supports a uniform, compositional theory of degree introduction (Wellwood 2012, 2014, 2015, forthcoming); (ii) it implies an explicitly measurement-theoretic functional vocabulary (cp. Sassoon 2010; cf. Krantz, Luce, Suppes \& Tversky 1971; Berka 1983; Roberts 1985); and (iii) it unifies otherwise disparate experimental results with children and adults (e.g., Gathercole 1985; Halberda \& Feigenson 2008; Odic, Pietroski, Hunter, Lidz \& Halberda 2013).

\section{Ambiguity + S-monotonicity}

The form of Ambiguity account that I consider interprets $\mathrm{MUCH}$ as a variable valued by measure functions. A constraint on the assignment of values to this variable captures the Monotonicity generalization directly ('S-monotonicity', after Schwarzschild 2002, 2006; see also Nakanishi 2007; Wellwood, Hacquard \& Pancheva 2012, and others). Additionally, MANY is interpreted as a cardinality function, which gets the Number generalization directly. I briefly sketch such an account, and then present some reasons to seek an alternative.

\subsection{Morphosyntax}

Characteristic of approaches to nominal comparatives since at least Bresnan 1973 that consider their occurrences with bare and plural nouns like (1) is the assumption that the form more is homophonous between two distinct morphosyntactic structures. In the context of a bare noun, more corresponds to the complex MUCH-ER, (5a), and in the context of a plural noun, it corresponds to MANY-ER, (5b).

(5) Ambiguity account: Morphosyntactic decomposition

a. more $_{1}$ coffee $\leadsto$ MUCH-ER COFFEE

b. more $_{2}$ coffees $\leadsto$ MANY-ER COFFEE-PL

Bresnan pointed out that more appears to belong to a semantically coherent category of functional items that usually overtly cue morphosyntactic complexity; for example, more coffee and as much coffee, which involve 'strictly greater than' and 'at least as great' relations between amounts of coffee, respectively (cf. Schwarzschild 2008). Given Bresnan's analysis, the compositional semantics can follow directly: both more and as much share the piece MUCH, which introduces 'amounts' or 'degrees' in relation to the coffee, in the present examples; and, they differ in the pieces -ER and AS, which introduce the relevant relations between degrees.

By the same reasoning, it is tempting to offer a distinct decompositional analysis of more when it occurs with plural nouns. Surveying a broader range of forms, it 
quickly becomes apparent that English includes a grammatical difference between the surface forms much and many, (6-7).

(6) Only much with bare nouns

a. as/too/so much coffee

b. * as/too/so many coffee

(7) Only many with plural nouns

a. * as/too/so much coffees

b. as/too/so many coffees

Moreover, there is an important semantic difference that attends the occurrences of these forms. Whenever many occurs overtly, the Number generalization holds. We would thus be missing a generalization if we simply talked in broad terms about comparisons between 'amounts', as we do with much, or as we would appear to do if we uniquely decomposed more in such terms. Thus, it has seemed reasonable both for distributional and semantic reasons to posit something like (5).

\subsection{Semantics}

An Ambiguity account of this sort must give two semantic analyses: one for more ${ }_{1}$, the surface realization of MUCH-ER, and one for more $_{2}$, i.e. MANY-ER. ${ }^{5}$

The basic observation about comparatives with bare nouns (those featuring more $_{1}$ ) is that they display variable dimensionality, both across and within predicates. The examples in (8) together illustrate both sorts of variability: combining more 1 with coffee allows for comparisons by volume or weight, as in (8a); and, on the assumption that non-cardinal dimensions signal the presence of more 1 as opposed to that of more $_{2}$, combining that expression with run allows for comparisons by distance or duration, as in (8b).

Variability within predicates
a. more $_{1}$ coffee
volume, weight
b. run more 1
distance, duration

Yet, it is not the case that more $_{1}$ is unconstrained. While its combination with coffee supports comparisons along a plurality of dimensions (e.g., (8a)), more 1 coffee cannot be understood as a comparison along many of the dimensions we

5 A complete treatment would also link the interpretations assigned to the pieces MUCH and MANY to their meaning contribution outside of the comparative forms; see e.g. Rett 2008; Solt 2015; and Wellwood 2014 for recent discussion. 
Structure preservation

might otherwise care to compare coffee along, e.g. temperature, tastiness, strength, etc.; (9a). Whatever accounts for this pattern of restricted variability, it cannot simply be that more $_{1}$ is unable to introduce such measures, since examples where it does can easily be constructed. For one, more ${ }_{1}$ global warming is most naturally interpreted as a comparison by temperature, (9b).

(9) Variability across predicates
a. more $_{1}$ coffee
volume, *temperature
b. more $_{1}$ global warming
temperature, *volume

Finally, the basic observation about comparatives with plural nouns (those, or so we are supposing, that feature more $_{2}$ ) is that they appear to only involve comparisons by cardinality. ${ }^{6}$ This is true both for cases where the head noun that has been pluralized would otherwise permit dimensions other than cardinality, (10), as well as for cases where the head noun fails to permit other dimensions for comparison, (11), or at least not as easily.

(10) Excludes dimensions available to $\mathrm{N}$
a. more $_{1}$ coffee
b. more $_{2}$ coffees

volume, *cardinality

cardinality, *volume

(11) Introduces dimension unavailable to $\mathrm{N}$

a. more traffic cone

b. more $_{2}$ traffic cones

cardinality

The sort of Ambiguity approach that I have sketched can straightforwardly account for these patterns. It need only interpret the piece $\mathrm{MUCH}$, realized in part by more $_{1}$, as expressing a contextually-provided measure function; this is represented in (12a), where $\sigma$ is any assignment function, and $\sigma(\mu)$ is the measure function that $\sigma$ assigns to $\mu$. In contrast, the piece MANY, realized in part by more $_{2}$, can be interpreted as a cardinality function - that is, any function that maps pluralities ( $x x$, $y y$, etc.) to the number of their individual parts, (12b).

(12) Ambiguity account: semantics of more $_{1}$ and more $_{2}$, respectively

a. $\quad \llbracket \mathrm{MUCH}_{\mu}-\mathrm{ER} \rrbracket^{\sigma}(d)(x)=\sigma(\mu)(x)>d$

b. $\llbracket \mathrm{MANY}-\mathrm{ER} \rrbracket^{\sigma}(d)(x x)=$ cardinality $(x x)>d$

6 For notes regarding a prominent potential class of counterexamples, see footnote 2 . 
This account therefore captures the Number generalization directly: we only observe the cardinal dimension with plural nouns because only MANY occurs with plurals. A little more needs to be said in order to capture the Monotonicity generalization. Building on Schwarzschild 2002, 2006, we get this by adding the condition that permissible values of $\mu$ given $\sigma$ must be $S$ (chwarzschild)-monotonic. ${ }^{7,8}$

$$
\begin{aligned}
& \text { S-monotonicity } \\
& \forall x, y \in D_{P} \text {, if } x \prec_{P} y \text {, then } \mu(x)<\mu(y) .
\end{aligned}
$$$$
\text { Schwarzschild 2002, } 2006
$$

In other words, permissible $\sigma(\mu)$ applied to some $x$ must be such that any strict ordering relations holding between $x$ and any $y$ in that same domain are preserved in the mapping to degrees.

To see what work this does, suppose along with many semanticists that the extension of coffee is a mereologically-ordered set of portions of coffee. ${ }^{9}$ More formally, assume a context in which $\llbracket c o f f e e \rrbracket=\left\{\ldots, c, c^{\prime}, c \oplus c^{\prime}, \ldots\right\}=D_{C}$, a set closed under sum, $\oplus$. There is a partial ordering on this set, $\preccurlyeq_{C}$, and, intuitively, for any $x, y \in D_{C}$ such that $x \prec_{C} y$, (13) hold. Given that temperature generally fails to preserve non-trivial part-whole relations, S-monotonicity prohibits selecting such a measure for expressions like more $_{1}$ coffee.

$$
\begin{array}{ll}
\text { a. } & \text { volume }(x)<\operatorname{volume}(y) \\
\text { b. } & \operatorname{weight}(x)<\operatorname{weight}(y) \\
\text { c. } & \text { temperature }(x) \nless \operatorname{temperature}(y) \\
\text { d. } & \text { etc. }
\end{array}
$$

This account, then, presents a clean picture of the facts that we have so far considered. Nonetheless, there are reasons to seek an alternative.

\subsection{Reasons to seek an alternative}

Cardinality meets Schwarzschild's condition, since the count of elements in a plurality will be greater than that of any of its proper subpluralities. However, the putative MANY that invokes cardinality isn't subject to that condition; its only role is to regulate the selection of measure functions with MUCH. On this picture, the apparent adherence to S-monotonicity in sentences like (1b) is accidental. If there

7 See also Nakanishi 2007; Wellwood et al. 2012; cp. Champollion 2010, 2017.

8 Note that the formulation I give in the text is more general than the original, which refers explicitly to mereological structure. This choice reflects my assumption that any given noun or noun phrase comes with a unique ordering on its domain, against which S-monotonicity is evaluated. This of course places a heavy burden on the ontology; see Wellwood 2015 for some discussion.

9 I also assume that such sets are anti-atomic; see Gillon 2012 for recent discussion and references. 
Structure preservation

\begin{tabular}{l|ll|l}
\hline language & volume & number & difference \\
\hline \hline English & much soup & many cookies & 'lexical' \\
\hline Spanish & mucha sopa & muchas galletas & agreement \\
Italian & molta minestra & moltíbiscotti & agreement \\
French & beaucoup de soupe & beaucoup de biscuits & N morphology \\
Macedonian & mnogu supa & mnogu kolaci & N morphology \\
Mandarin & henduo tang & henduo kuai quqi & classifier \\
Bangla & onek sup & onek-gulo biskuT & classifier
\end{tabular}

Table 2 Where many signals number with plural Ns in English, other languages combine a univocal form with (broadly) plural marking. (See Wellwood 2014 for attributions of these data to native speaker consultants.)

are reasons to doubt the existence of primitive MANY, though, and furthermore to suppose that comparatives always involve $\mathrm{MUCH}$, then it must be that cardinality is a required value for $\mu$ under certain conditions.

I see two reasons for doubting the existence of MANY. One is crosslinguistic, while the second is more theory-internal.

First, surveying an array of languages, there is no supporting evidence for the suggestion that there are two distinct primitives in the relevant semantic field. That is, the English distinction between much and many does not recur: instead, the shift from consideration of (e.g.) volume to number is reflexive on the presence of (broadly) plural marking, with no change in the form of the expression that introduces the measure; see Table 2. Spanish transparently illustrates the general pattern: speaking of Juan, we can say that he drank a lot of beer (by volume) using the form in (14a), or that he drank a lot of beers (by number) using (14b).

Spanish: Univocal form, unequivocal semantics

a. mucha cerveza

volume

b. muchas cervezas

number

Second, if only S-monotonicity restricts the selection of values for $\mu$, then the account will overgenerate: so far, it can't guarantee comparisons by number for more $_{1}$ where they're observed. This is reportedly the case for comparatives with superordinate mass nouns like furniture (see Bale \& Barner 2009 for discussion and references), ${ }^{10}$ (15a). The comparison by number is restricted to contexts with

10 Barner \& Snedeker (2005) report experimental data supporting this: presented with pictures of small sets of furniture and asked which is "more", both adults and children strongly preferred to answer based on the number of pieces. Grimm \& Filip (2017) suggest that such results do not reflect a 
Wellwood

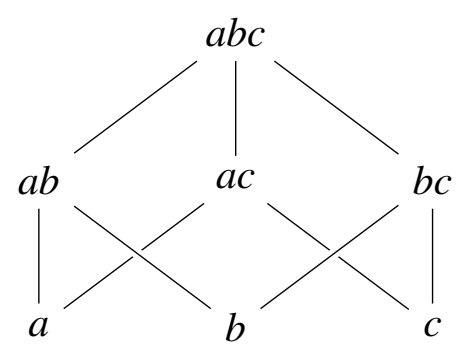

Figure 1 Hypothetical extension for furniture in a context. Nodes represent pieces of furniture and pluralities thereof, lines represent plural-part relations.

the bare noun, compare (15a) and (15b); indeed, the comparatives with as here obligatorily involve much, (16).

a. Ann bought more furniture than Bill did. cardinality, *weight, ...

b. * Ann bought more furnitures than Bill did.

$?$ ?

a. Ann bought as much furniture as Bill did. cardinality, *weight, ...

b. * Ann bought as many furniture(s) as Bill did.

Here's the issue. Let the extension of furniture be as in Figure 1; it is a join semi-lattice with atomic minimal parts $a, b$, and $c$ (e.g., a table, chair, ...). Formally, this extension shares many of the formal properties typically assumed for plural nouns. Whichever plurality of pieces $x x, y y$ we pick such that $x x \prec y y$, it will be true that weight $(x x)<\operatorname{weight}(y y)$. Given that this is so, and given that we are armed so far only with S-monotonicity, we should expect that (15a) could be interpreted as a comparison of weights, contrary to fact. I call this 'the weight problem'.

The crosslinguistic picture suggests a lack of support for primitive MANY from the perspective of morphology. The weight problem suggests a lack of support for

semantic obligation, however. These authors presented adults with text-based descriptions of two sets of (e.g.) pieces of furniture, and found a substantial proportion of non-number-based responses. For example, their Experiment 4 varied whether the furniture's utility was relevant, before asking the comparative question. When use was not relevant, judgments indeed tracked the number of pieces (75\% number-based). When use was relevant, however, the 'heterogeneity' of the two sets better predicted participants' judgments (65\% heterogeneity-based). In ongoing work in my lab, we systematically varied the number, size, and heterogeneity (operationalized in a couple of different ways) of two sets of furniture in pictures, and have found that both adults and children strongly preferred to judge based on number (adults 92\% number-based; children, $72.1 \%$ number-based). We will see if we can reproduce Grimm \& Levin's effect using contextual manipulations. If we can, this could support their analysis in which furniture-type nouns introduce both individuals and events into the semantics. Given the availability of a different 'thing' to measure, it should be possible to render such an account consistent with the ontology-dependent theory I advance here. 
Structure preservation

such a primitive from the perspective of semantics, as well, at least if MUCH can be restricted to cardinality measures for nouns like furniture and coffees.

\section{Univocality + S-monotonicity + A-invariance}

The Univocality account aims to eliminate the need for a primitive MANY by constraining the semantics of $\mathrm{MUCH}$. On this account, more univocally realizes $\mathrm{MUCH}-$ $\mathrm{ER}$, and the value $\sigma(\mu)$ introduced by MUCH is constrained by S-monotonicity as well as by a constraint that I will call A(utomorphism)-invariance. Where Smonotonicity ensures that non-trivial ordering relations are preserved in the mapping to degrees, A-invariance ensures that the mapping is invariant with respect to any strongly structure-preserving permutation on the measured domain.

I first point to the required morphophonological component of any univocal account - an analysis in which many is an allomorph of MUCH (cf. Spanish (14)). I focus, however, on developing a semantics for MUCH that can restrict comparatives involving coffees, ideas, and furniture to just comparisons by number.

\subsection{Morphosyntax}

Recall the data in (6) and (7): only much can surface with bare nouns, while only many can surface with plural nouns (e.g., *as many coffee, *as much coffees). The Univocality account will suppose that this is a surface difference arising from the morphophonological component of grammar. I thus assume, for now, the existence of any sufficient such rule that will produce many where it occurs - that is, in the environment of the nominal plural, e.g. (17).

$$
\mathrm{MUCH} \rightarrow \text { morph many / _ PL }
$$

Of course, any serious proposal of this type will have to specify in greater detail the appropriate syntactic scope and limits of the relevant rule; we will want to generate as many tall books, for example, but not *as much tall books, etc. I do not attempt to do this here; the reader may see Dunbar \& Wellwood's (2016) updated fragment of Bresnan's 1973 morphosyntax, for a place to begin thinking about this.

\subsection{Semantics}

If more uniquely realizes MUCH-ER, then just one semantic analysis is needed: the semantics must guarantee that $\sigma(\mu)$ is valued by a cardinality function whenever MUCH targets nouns like coffees and furniture.

As discussed extensively by Bale \& Barner (2009), the critical connection between these types of nouns is that their domains consist of pluralities composed 
of individuals, the entities we typically refer to as 'atoms' (thus, both nouns have domains with the structure of atomic join semi-lattices). Bale \& Barner's accounting of the Number generalization capitalizes on this formal similarity, but the restriction that they impose on (non-decomposed) more is, so far, essentially arbitrary. ${ }^{11}$

$$
\begin{aligned}
& \text { a. I bought more coffees. } \\
& \text { b. I bought more furniture. }
\end{aligned}
$$

cardinality, *weight cardinality, *weight

We want a principled account in which the constraint on the selection of measure functions accords with the following intuition: domains $D_{P}$ that can only be measured by cardinality can only be so measured because only cardinality uniquely characterizes - or represents- $D_{P}$. To make the intuition clear, consider that cardinality assigns all of the singletons in a plural domain $D_{P}$ (assuming an inclusive theory of plurality) to the number 1 , pluralities consisting of two atoms to the number 2 , etc. In contrast, weight can assign different values to any of these, such that a given singleton could weigh the same as a doubleton, etc.

To eliminate the weight problem, then, I propose that the selection of values for $\sigma(\mu)$ is constrained not only by S-monotonicity but by A(utomorphism)Invariance. ${ }^{12} \mathrm{~A}$-invariance says that permissible $\mu$ s assign the same value to any $x \in D_{P}$ as to $h(x) \in D_{P}$, where $h$ is a strongly structure-preserving permutation of $D_{P}$, as defined directly.

\section{A-invariance}

$$
\forall x \in D_{P}, \forall h \in A u t\left(\left\langle D_{P}, \precsim_{P}\right\rangle\right), \mu(x)=\mu(h(x))
$$

More formally, I assume that an automorphism $h$ is any bijective function that maps a set, here $D_{P}$, to itself, in accord with (19): any ordering relations holding between $x$ and $y$ in $D_{P}$ must hold between $h(x)$ and $h(y)$. Since any automorphism $h$ on $D_{P}$ is invertible (bijectivity), and its domain is (exactly) the same as its range (endomorphy), (19) cannot be satisfied by a function that preserves only trivial ordering relations between elements of $D_{P}$ (order preservation).

$$
\forall x, y \in D_{P}, x \precsim_{P} y \text { iff } h(x) \precsim_{P} h(y)
$$

Some quick examples should make this clear. Suppose here and below that $D_{P}=\{a, b, c, a b, a c, b c, a b c\}$ (the inclusive set of pluralities whose minimal parts

11 The account goes roughly like this: resolving the variable dimensionality evinced by more involves an orderly selection from a list of measure functions; and, cardinality holds a privileged position in this list; if the comparison targets entities in an atomic semi-lattice, there is a requirement that the selection stop at this position.

12 I consider the question, below, whether both constraints are needed, or whether A-invariance could be sufficient for capturing our two generalizations. My tentative conclusion, there, is negative. 
are the individuals $a, b$, and $c$ ), and the ordering $\precsim P$ on this set has all of the properties that we think the domains of plural nouns like toys or superordinate mass nouns like furniture have (i.e., they are atomic join semi-lattices).

Now then, $h$ in (20) is an example of an automorphism on $D_{P}$.

Automorphism $h$ in $A u t\left(\left\langle D_{P}, \precsim_{P}\right\rangle\right)$

a. $h=[a \mapsto b, b \mapsto c, c \mapsto a, a b \mapsto b c, a c \mapsto a b, b c \mapsto a c, b c \mapsto a b c]$

b. range $(h)=$ domain $(h)$

[endomorphy]

c. there is a function $g$ such that $\operatorname{domain}(g)=\operatorname{range}(h)$

[bijectivity]

d. $\neg \exists x, y\left[x \precsim P y \wedge h(x) \mathscr{L}_{P} h(y)\right]$

[order preservation]

There are many functions $h$ that are not automorphisms on $D_{P} ;(21)$ gives some examples, along with reasons for their failure.

(21) Functions $h$ not in $\operatorname{Aut}\left(\left\langle D_{P}, \precsim P\right\rangle\right)$

a. Any $h=[a \mapsto d, \ldots]$, since $d \notin D_{P}$

[not endomorphic]

b. Any $h=[a \mapsto b, c \mapsto b, \ldots]$, since not invertible

[not bijective]

c. Any $h=[a \mapsto c, a b \mapsto a, \ldots]$, since $a \precsim_{P} a b$, but $h(a) \mathscr{L}_{P} h(a b)$

[not order-preserving]

\subsection{No more weight problem}

Since any automorphism $h$ on atomic $\left\langle D_{P}, \succsim_{P}\right\rangle$ pairs singletons with singletons, doubletons with doubletons, etc., then any $x x \in D_{P}$ is such that cardinality $(x x)=$ cardinality $(h(x x))$, whatever else can be said about $D_{P}$. Therefore, measures by cardinality are A-invariant with respect to such a domain.

However, measures by weight are not; a counter-example is given in (22).

(22) Let $D_{P}=\{b, c, b c\}, h$ an automorphism on $D_{P}$ such that $h(b)=c$, and weight : $[b \mapsto 120 \mathrm{lbs}, c \mapsto 240 \mathrm{lbs}, \ldots]$. Then, since
a. weight $(h(b))=$ weight $(c)$,
b. weight $(h(b))=240 \mathrm{lbs}$; so,
c. weight $(h(b)) \neq$ weight $(b)$, because
d. $120 \mathrm{lbs} \neq 240 \mathrm{lbs}$.

Thus, an account that constrains the selection of measure functions for $\mathrm{MUCH}$ by A-invariance seems promising: it permits cardinality but excludes (at least in the general case) weight. Such an account also raises new questions. For example, 
(i) will A-invariance apply correctly outside of relevantly plural domains? And, (ii) should we understand A-invariance to supplement S-monotonicity, or could it replace that condition?

For now, my answers to these questions are tentative. Towards (i), I say 'yes'. If A-invariance applies whenever a measure function is considered as a value for $\mu$, then it must permit volume and weight with more coffee but exclude temperature, etc. And I think it does this. Suppose that the extension of coffee is a dense ordering of portions of coffee by inclusion. Any automorphism (hence, any $h \in A u t\left(\left\langle D_{C}, \succsim_{C}\right\rangle\right)$ ) will preserve this structure exactly. Indeed, it seems that just in the same way that cardinality can be said to represent essential structure of plural part-of relations, volume or weight do the same for material part-of relations.

Towards (ii), I suggest that the answer is 'no'. There are conceivable measure functions (i.e., functions that map individuals or events to degrees) that will fail to strongly preserve the structure of the domain for measurement, but which would satisfy A-invariance. Consider a hypothetical such function, one, that maps everything to the number 1 . This function trivially satisfies A-invariance, since any $x \in D_{P}$ will be such that one $(x)=1$, and of course one $(h(x))=1$, etc. Such a function will not satisfy S-monotonicity, however, since for any $x, y \in D_{P}$ such that $x \prec_{P} y$, it is not the case that one $(x)<$ one $(y)$.

If these answers are on the right track, then we have successfully subsumed our two generalizations to just one-Monotonicity. The difference between the sentences in (1) reflects the differing outcomes of calculating S-monotonicity and A-invariance against domains with different formal properties.

\subsection{Compositional sketch}

For completeness, this section briefly sketches a compositional implementation of the semantics. I demonstrate this for (1a) and (1b), repeated as in (23a) and (23b).

a. Ann bought more coffee than Bill did.

b. Ann bought more coffees than Bill did.

The lexical axiom for MUCH can be seen as the same on the two accounts that we have considered; in (24a), $\alpha$ indicates neutrality between portions, individuals, pluralities, events, etc. Correspondingly, -ER is interpreted as in (24b), where $\delta$ is the degree provided by a than-clause, when present. Combining these two by Functional Application as in (25), the result is a property of entities $\alpha$, the $\sigma(\mu)$-measure of which is greater than $\delta$.

$$
\begin{aligned}
& \text { a. } \llbracket \mathrm{MUCH}_{\mu} \rrbracket^{\sigma}=\lambda \alpha \cdot \sigma(\mu)(\alpha) \\
& \text { b. } \llbracket \mathrm{ER} \rrbracket^{\sigma}(\delta)=\lambda g \cdot \lambda \alpha \cdot g(\alpha)>\delta
\end{aligned}
$$


Structure preservation

\begin{tabular}{|l|llll|}
\hline language & base $\mathbf{N}$ & \multicolumn{3}{|c|}{ singular N } \\
\hline Russian & lyod & 'ice' & l'dina & 'block of ice' \\
Breton & geot & 'grass' & geot-enn & 'blade of grass' \\
Classical Arabic & teen & 'mud' & teenah & 'a chunk of mud' \\
Fox & owiiyaasi & 'meat' & owiiyaasa & 'a piece/cut of meat' \\
\hline
\end{tabular}

Table 3 Singulatives. (Based on Mathieu 2012 and references therein.)

\begin{tabular}{|l|lll|}
\hline language & base N & singular N & plural N \\
\hline Hebrew & se'ar 'hair' & sa'ar-a & sa'ar-ot \\
Breton & buzhug 'worms' & buzhug-enn & buzhug-enn-oú \\
Fox $^{14}$ & zhooniyaahi 'silver, money' & zhooniyaaha & zhooniyaaha-ki \\
Ojibwe $^{15}$ & mikwam 'ice' & mkwamiins & mkwamiins-ag \\
\hline
\end{tabular}

Table 4 Pluralized singulatives. (Based on Mathieu 2012 and references therein.)

$$
\llbracket \mathrm{ER} \rrbracket^{\sigma}(\delta)\left(\llbracket \mathrm{MUCH}_{\mu} \rrbracket^{\sigma}\right)=\lambda \alpha . \sigma(\mu)(\alpha)>\delta
$$

FA

(23a) and (23b) obviously overlap in their head noun, coffee, but they are evaluated along different dimensions. The way that $\mathrm{MUCH}$ works is that 'what is measured' and 'how it's structured' play an important role in calculating that dimensionality. I encode the overlap and difference, then, between coffee and coffees as a mapping between different (sub-)domains: bare coffee introduces a property of portions of coffee, while coffees introduces a property of pluralities, each atomic part of which is constituted by some coffee. On this view, pluralizing a noun like coffee involves mapping an anti-atomic domain to an atomic one.

A covert morpheme SG can be used to bridge the substance and plurality domains following, for example, Mathieu's (2012) work on singulatives. Such a morpheme is overt in other languages (see Table 3), and surfaces when substance-denoting nouns are pluralized (see Table 4). In other words, the derivation of coffees in English parallels Mathieu's analysis of Ojibwe 'icicles' (Figure 2). Semantically, we can assign SG the role of introducing a map from a property of substance to a property of atomic entities constituted by some of that substance. ${ }^{13}$

More formally, let's suppose that COFFEE introduces a property of portions of

13 A more theory-internal reason for taking this circuitous route-mapping a substance property to an atomic property to a plural property - is that it wouldn't do to map coffee simply to a property of atoms, to the exclusion of pluralities; such properties are not measurable (see Hackl 2001; Nakanishi 2007; Wellwood et al. 2012, etc). And indeed, according to Mathieu (p.c.), singulative-marked NPs in the relevant languages will not be available to comparative quantification, while those bearing singulative and plural will be. 
Wellwood

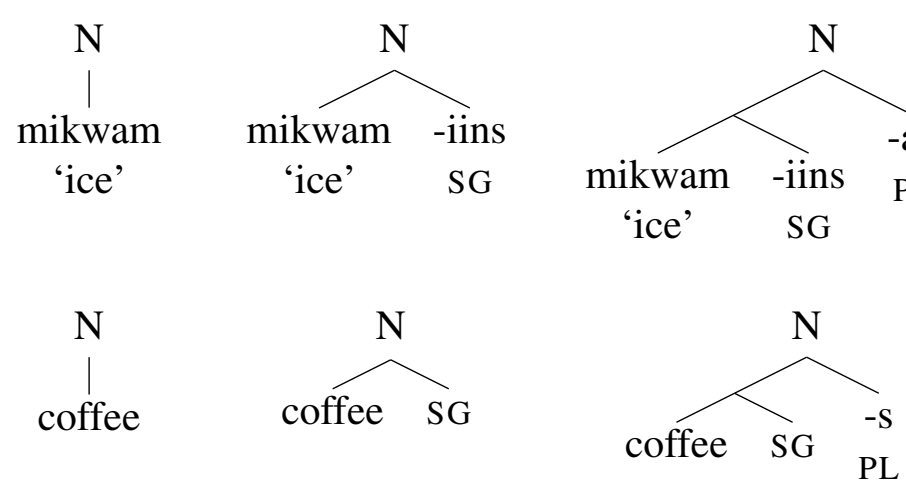

Figure 2 A parallel derivation for Ojibwe mikwamiinsag ('icicles', top; Mathieu 2012), and English coffees (bottom).

coffee $c$, (26a). SG maps such a property to a property of atomic entities that are materially constituted by some $c,(26 \mathrm{~b}) .{ }^{16}$ Issues of context-dependent atomicity are likely to be relevant here (see, e.g., Sutton \& Filip 2016 for recent discussion and references), so let us suppose that the context provides some salient individuation criteria-i.e., a value for a $C$ variable on atom in (26b). The semantics of PL, in turn, presupposes an individuated property, $f$ in (26c), and maps $f$ to a property of pluralities $x x$, every atomic part of which satisfies $f .^{17}$

a. $\llbracket \mathrm{COFFEE} \rrbracket^{\sigma}=\lambda x \cdot \operatorname{coffee}(x)$

b. $\llbracket \mathrm{SG} \rrbracket^{\sigma}=\lambda f: \operatorname{anti-atomic}(f) \cdot \lambda y: \operatorname{atom}_{C}(y) \cdot \exists x\left[f(x) \& y \triangleright^{m} x\right]$

c. $\llbracket \mathrm{PL} \rrbracket^{\sigma}=\lambda f: \operatorname{atomic}(f) \cdot \lambda x x \cdot \forall x[x x(x) \rightarrow f(x)]$

Thus, the semantic effect of combining COFFEE and SG is as in (27a), and combining that result with PL is as in (27b).

a. $\llbracket \mathrm{SG} \rrbracket^{\sigma}\left(\llbracket \mathrm{COFFEE} \rrbracket^{\sigma}\right)$

$$
=\lambda y: \operatorname{atom}(y) \cdot \exists x\left[\operatorname{coffee}(x) \& y \triangleright^{m} x\right]
$$

b. $\llbracket \mathrm{PL} \rrbracket^{\sigma}\left(\llbracket \mathrm{SG} \rrbracket^{\sigma}\left(\llbracket \mathrm{COFFEE} \rrbracket^{\sigma}\right)\right)$

[abb. as coffees below]

$=\lambda x x . \forall y: \operatorname{atom}(y)\left[x x(y) \rightarrow \exists x\left[\operatorname{coffee}(x) \& y \triangleright^{m} x\right]\right]$

As desired, combining these NPs with more means that constraints on the selection of $\sigma(\mu)$ will be calculated relative to its application to different things: it

16 See Parsons 1979 and Link 1983, for example.

17 In (26c) and below, I write $x x(x)$ to remain neutral between a variety of options, depending on one's theory of the representation of plural properties. For example, it could mean $x \in x x$ if $x x$ ranges over sets, among $(x, x x)$ if $x x$ is a plural variable, or $x \succcurlyeq x x$ if pluralities are sums, etc. For different approaches, see for example Link 1983; Boolos 1984; Winter 2001. 
Structure preservation

will be relativized to a partial ordering on portions of coffee in (28a), and to a partial ordering on pluralities in (28b).
a. $\llbracket$ more $_{\mu}$ coffee $\rrbracket^{\sigma}=\lambda x$.coffee $(x) \& \sigma(\mu)(x)>\delta$
PM
b. $\llbracket$ more $_{\mu}$ coffees $\rrbracket^{\sigma}=\lambda x x \cdot \operatorname{coffees}(x x) \& \sigma(\mu)(x x)>\delta$
$\mathrm{PM}$

I interpret the sentences in (1a)/(23a) and (1b)/(23b) as in (29a) and (29b), respectively. Each expresses an existential statement about buying events by Ann, and differ just in what is said about their themes: it is some portion of coffee measuring greater than any corresponding portion for Bill, (29a), or some plurality of coffees measuring greater than any corresponding plurality for Bill. ${ }^{18}$

a. $\exists e[\operatorname{ag}(e, a) \& \operatorname{buying}(e) \& \exists x[\operatorname{th}(e, x) \& \operatorname{coffee}(x) \& \sigma(\mu)(x)>\delta]]$, where $\delta=$ $\max (\lambda d . \exists e[\operatorname{ag}(e, b) \&$ drinking $(e) \& \exists x[\operatorname{coffee}(x) \& \operatorname{th}(e, x) \& \sigma(\mu)(x) \geq d]])$

b. $\exists e\left[\operatorname{ag}(e, a) \& \operatorname{buying}(e) \& \exists x x\left[\operatorname{th}(e, x x) \& \operatorname{coffees}(x x) \& \sigma(\mu)(x x)>\delta^{\prime}\right]\right]$, where $\delta^{\prime}=$ $\max (\lambda d . \exists e[\operatorname{ag}(e, b) \&$ drinking $(e) \& \exists x x[\operatorname{coffees}(x x) \& \operatorname{th}(e, x x) \& \sigma(\mu)(x x) \geq d]])$

\section{Beyond truth conditions}

So far, I have provided conceptual, empirical, and theoretical reasons to support a univocal account of the structure and meaning of comparatives with more. Now, I briefly recall a small set of experimental results that suggest the same conclusion. I understand these results to relate coherently and straightforwardly to the representational theory offered under a Univocality account, whereas they do not hang together quite so well on an Ambiguity account.

For example, all else equal Univocality expects the acquisition trajectories of much and many in English to intertwine, but Ambiguity doesn't. And in particular, if many is derived from MUCH by rule as I have suggested, then we might expect that relation to involve overgeneralizing the form much, rather than the other way around. This is in fact the pattern that Gathercole 1985 observes: children up to 7 years 6 months overextended much with plural nouns, but they never used many with bare nouns. This pattern is reminiscent of other error patterns familiar from morphological acquisition, and unsurprising if Univocality is right.

Moreover, Univocality expects simultaneous acquisition of more with bare and plural occurrences (again, all else equal). And so far, the evidence is consistent with this sort of 'all or nothing' acquisition. Children appear to acquire more with its sensitivity to measurement of substances versus pluralities at the same time, as revealed by studies examining how that understanding develops (Odic et al. 2013).

18 For space reasons, I can't provide the full derivational details. Similar compositional assumptions are worked out in detail in Wellwood 2014, though. 
Part of this, of course, is an adult-like sensitivity to how grammatical context can shift the dimension for $\mathrm{N}$, regardless of what one otherwise prefers to think about when given more $N$ : given an 'ambiguous' world supporting both volume and number quantification, children chose volume when asked about "more fem", but number when asked about "more fems" (Barner \& Snedeker 2006).

For a last point, consider two facts that a semantics for comparatives might want to address: (i) children's earliest evaluation of plural nominal comparatives uses their Approximate Number System, or ANS (Dehaene 1997, Feigenson, Dehaene \& Spelke 2004, Halberda \& Feigenson 2008), and their ability to recruit this system to answer a "more" question is independent of their understanding of natural number (Halberda, Taing \& Lidz 2008); (ii) adults, too, use their ANS to answer "more" questions, at least under speeded conditions (cf. Pietroski, Lidz, Hunter \& Halberda 2009, Lidz, Halberda, Pietroski \& Hunter 2011, cp. Kotek, Sudo \& Hackl 2015). ${ }^{19}$

Supposing Ambiguity, recruiting the ANS in the evaluation of a plural nominal comparative as in (i) and (ii) above would have to be explained away as reflecting some kind of partial or imperfect knowledge about what such sentences mean. ${ }^{20}$ In contrast, so long as the ANS 'scale' meets the conditions I've laid out for the selection of measure functions given relevantly plural domains (i.e., so long as it is S-monotonic and A-invariant here), a measure function like approximate number would in fact be grammatically licensed.

Does this point go in favor of Univocality, then? I can offer a couple of thoughts. ANS representations have a richer structure than exact number representations, but they might not be relevantly different enough such that ANS representations could fail to meet our conditions; Gallistel \& Gelman (1992) argue at length that the systems are in fact isomorphic. On the face of it, then, finding a relevant counterexample should be difficult. Meanwhile, this question invites consideration of what we might want to say about languages with a near translational equivalent of more, but limited access to exact number (cf. Pica, Lemer, Izard \& Dehaene 2004).

The two accounts thus plausibly differ in how they would characterize successful grasp of the meaning of comparative sentences. They definitely differ in the aptness of their predictions as to how the acquisition of that meaning should proceed, and as to how it should come packaged across languages. All else being equal, a semantic theory with a better shot at predicting relevant facts in neighboring domains should be preferred over its alternatives.

19 Some of these results involve evaluating most sentences. most shows the same variability and constraints as more, and some recent analyses analyze the superlative as constructed out of the comparative (e.g. Stateva 2003, Bobaljik 2012, Szabolcsi 2012, Dunbar \& Wellwood 2016).

20 That is, so long as one doesn't modify the Ambiguity account so that MANY contains a variable over relevantly number-like measure functions. I don't know that anyone would seriously entertain such an account, though, so I won't either. 
Structure preservation

\section{Conclusion}

I argued that more uniquely decomposes into MUCH and -ER. I suggested a semantics for MUCH designed to capture central facts about how sentences with more are understood: the dimension for comparison in any given comparative can vary, but only within the limits prescribed by coupling strong structure preservation with differences in the measured domain.

By emphasizing structure preservation as critical to this understanding, the account invites measurement-theoretic notions into the functional vocabulary. Measurement is generally understood as involving a structure-preserving map between an observed or observable relation between entities, and mathematical structures that represent the quantitative differences inherent in those relations (e.g. Berka 1983). Meanwhile, the mass/count literature tends to suppose that expressions like coffee and coffees apply to entities with different properties, albeit similar relational structures. If my theory is correct, then the differences in dimensionality observed with these expressions is a consequence of those differences.

Measurement-theoretic notions have been invoked in semantic theory before. Recently, Sassoon 2010 uses the formal typology of scales from measurement theory (e.g., ordinal, ratio, interval, etc.) to categorize the scales associated with gradable adjectives and scalar modifiers. Lassiter 2011 does similar in his analysis of modal auxiliaries and attitude verbs like want. I see my project as complementary. For one thing, the comparatives proper seem importantly different from degree modifiers; see Fults 2006. For another, my account invokes the concept of measurement, rather than selections from a typology of its instances.

A push for univocality in one empirical corner invites the question of how much further it should be pushed. In general, Ambiguity-inclined theorists appear to be okay with the assumption that the more occurring with coffee is different from that occurring with intelligent: more introduces measure functions in the former case, but intelligent does so in the latter. Comparing these nominal and adjectival instances, though, it seems that here too the evidence for ambiguity might be found wanting (see Wellwood 2012, 2014, forthcoming, Dunbar \& Wellwood 2016, Cariani et al. 2018; submitted for attempts to extend Univocality in this direction).

\section{References}

Acquaviva, Paolo. 2008. Lexical plurals: A morphosemantic approach. Oxford Studies in Theoretical Linguistics. Oxford, UK: Oxford University Press.

Bale, Alan \& David Barner. 2009. The interpretation of functional heads: Using comparatives to explore the mass/count distinction. Journal of Semantics 26(3). 217-252. 
Barner, David \& Jesse Snedeker. 2005. Quantity judgments and individuation: evidence that mass nouns count. Cognition 97(1). 41-66. doi:10.1016/j.cognition.2004.06.009.

Barner, David \& Jesse Snedeker. 2006. Children's early understanding of mass-count syntax: individuation, lexical content, and the number asymmetry hypothesis. Language Learning and Development 2. 163-194. doi:10.1207/s154733411ld0203_2.

Berka, Karel. 1983. Measurement: its Concepts, Theories, and Problems, vol. 72 Boston Studies in the Philosophy and History of Science. Dordrecht, Holland: D. Reidel Publishing Company.

Bhatt, Rajesh \& Roumyana Pancheva. 2004. Late merger of degree clauses. Linguistic Inquiry 35(1). 1-46. doi:10.1162/002438904322793338.

Bobaljik, Jonathan David. 2012. Universals in comparative morphology: Suppletion, superlatives, and the structure of words. Boston MA: MIT Press.

Boolos, George. 1984. To be is to be the value of a variable (or some values of some variables). Journal of Philosophy 81. 430-450.

Bresnan, Joan. 1973. Syntax of the comparative clause construction in English. Linguistic Inquiry 4(3). 275-343.

Cariani, Fabrizio, Paolo Santorio \& Alexis Wellwood. 2018; submitted. Confidence reports. Northwestern University Ms. http://www.philarchive.org/rec/CARCR-7.

Champollion, Lucas. 2010. Parts of a whole: Distributivity as a bridge between aspect and measurement. Philadelphia, PA: University of Pennsylvania dissertation.

Champollion, Lucas. 2017. Parts of a whole: Distributivity as a bridge between aspect and measurement. Oxford Studies in Theoretical Linguistics. Oxford, UK: Oxford University Press.

Cresswell, Max J. 1976. The semantics of degree. In Barbara Hall Partee (ed.), Montague grammar, 261-292. New York: Academic Press.

Dehaene, Stanislas. 1997. The number sense: How the mind creates mathematics. New York, NY: Oxford University Press.

Dunbar, Ewan \& Alexis Wellwood. 2016. Addressing the 'two interface' problem: The case of comparatives and superlatives. Glossa: a journal of general linguistics 1(1). 5.1-29. doi:10.5334/gjgl.9.

Feigenson, Lisa, Stanislas Dehaene \& Elizabeth Spelke. 2004. Core systems of number. Trends in Cognitive Science 8(7). 307-314. doi:10.1016/j.tics.2004.05.002.

Fults, Scott. 2006. The structure of comparison: An investigation of gradable adjectives. College Park, MD: University of Maryland dissertation.

Gallistel, Charles R. \& Rochel Gelman. 1992. Preverbal and verbal counting and computation. Cognition 44. 43-74. doi:10.1016/0010-0277(92)90050-R.

Gathercole, Virginia C. 1985. More and more and more about "more". Journal of 
Structure preservation

Experimental Child Psychology 40. 73-104. doi:10.1016/0022-0965(85)900669.

Gillon, Brendan S. 2012. Mass terms. Philosophy Compass 7(10). 712-730. doi:10.1111/j.1747-9991.2012.00514.x.

Grimm, Scott \& Hana Filip. 2017. Furniture and other artifactual aggregates. University of Rochester Ms.

Hackl, Martin. 2001. Comparative quantifiers and plural predication. In K. Megerdoomian \& Leora Anne Bar-el (eds.), West coast conference of formal linguistics (WCCFL) XX, 234-247. Somerville, Massachusetts: Cascadilla Press.

Halberda, Justin \& Lisa Feigenson. 2008. Developmental change in the acuity of the "number sense": The approximate number system in 3-, 4-, 5-, and 6-year-olds and adults. Developmental Psychology 44(5). 1457-1465.

Halberda, Justin, Len Taing \& Jeffrey Lidz. 2008. The development of "most" comprehension and its potential dependence on counting ability in preschoolers. Language Learning and Development 4(2). 99-121. doi:10.1080/15475440801922099.

Heim, Irene. 1985. Notes on comparatives and related matters. University of Texas, Austin Ms.

Heim, Irene. 2001. Degree operators and scope. In Caroline Fery \& Wolfgang Sternefeld (eds.), Audiatur vox sapientiae. a festschrift for Arnim von Stechow, 214-239. Berlin: Akademie Verlag.

Kennedy, Chris. 1999. Projecting the adjective: The syntax and semantics of gradability and comparison. New York: Garland.

Kotek, Hadas, Yasutada Sudo \& Martin Hackl. 2015. Experimental investigations of ambiguity: the case of most. Natural Language Semantics 23. 119-156. doi:10.1007/s11050-015-9113-0.

Krantz, D.H., R.D. Luce, P. Suppes \& Amos Tversky. 1971. Foundations of measurement, vol. I: Additive and polynomial representations. New York: Academic Press.

Lassiter, Daniel. 2011. Measurement and modality: The scalar basis of modal semantics. New York, NY: New York University dissertation.

Lidz, Jeffrey, Justin Halberda, Paul Pietroski \& Tim Hunter. 2011. Interface transparency and the psychosemantics of most. Natural Language Semantics 6(3). 227-256. doi:10.1007/s11050-010-9062-6.

Liebesman, David. 2016. Counting as a type of measuring. Philosopher's Imprint 16(12). 1-25.

Link, Godehard. 1983. The logical analysis of plurals and mass terms: A latticetheoretical approach. In Rainer Bäuerle, Christoph Schwarze \& Arnim von Stechow (eds.), Meaning, use and interpretation of language, 302-323. Berlin, Germany: DeGruyter. 
Mathieu, Éric. 2012. Flavors of division. Linguistic Inquiry 43(4). 650-679.

Nakanishi, Kimiko. 2007. Measurement in the nominal and verbal domains. Linguistics and Philosophy 30. 235-276. doi:10.1007/s10988-007-9016-8.

Odic, Darko, Paul Pietroski, Tim Hunter, Jeffrey Lidz \& Justin Halberda. 2013. Young children's understanding of "more" and discrimination of number and surface area. Journal of Experimental Psychology: Learning, Memory, and Cognition 39(2). 451-461.

Parsons, Terence. 1979. An analysis of mass terms and amount terms. In Francis Jeffrey Pelletier (ed.), Mass Terms: Some Philosophical Problems, 137-166. Dordrecht.

Pica, Pierre, Cathy Lemer, Véronique. Izard \& Stanislas Dehaene. 2004. Exact and approximate arithmetic in an Amazonian indigene group. Science 306(5695). 499-503. doi:10.1126/science.1102085.

Pietroski, Paul, Jeffrey Lidz, Tim Hunter \& Justin Halberda. 2009. The meaning of most: semantics, numerosity, and psychology. Mind \& Language 24. 554-585. doi:10.1111/j.1468-0017.2009.01374.x.

Rett, Jessica. 2008. Degree modification in natural language. New Brunswick, NJ: Rutgers dissertation. doi:10.7282/T3WH2Q8C.

Roberts, Fred S. 1985. Measurement theory: with applications to decisionmaking, utility, and the social sciences, vol. 7 Encyclopedia of mathematics and its applications. Cambridge: Cambridge University Press.

Sassoon, Galit Weidman. 2010. Measurement theory in linguistics. Synthese 174. 151-180. doi:10.1007/s11229-009-9687-5.

Schwarzschild, Roger. 2002. The grammar of measurement. In B. Jackson (ed.), Semantics and Linguistic Theory (SALT) XII, 225-245. Cornell University, Ithaca, NY: CLC Publications. doi:10.3765/salt.v12i0.2870.

Schwarzschild, Roger. 2006. The role of dimensions in the syntax of noun phrases. Syntax 9(1). 67-110. doi:10.1111/j.1467-9612.2006.00083.x.

Schwarzschild, Roger. 2008. The semantics of comparatives and other degree constructions. Language and Linguistics Compass 2(2). 308-331. doi:10.1111/j.1749-818X.2007.00049.x.

Schwarzschild, Roger. 2012. NeoNeoNeo Davidsonian Analysis of Nouns. Handout for the 2nd Mid-Atlantic Colloquium for Studies in Meaning, University of Maryland.

Seuren, Pieter A. M. 1973. The comparative. In F. Kiefer \& N. Ruwet (eds.), Generative Grammar in Europe, 528-564. Dordrecht: D. Reidel Publishing Company.

Seuren, Pieter A. M. 1984. The comparative revisited. Journal of Semantics 3. 109-141. doi:10.1093/jos/3.1-2.109.

Solt, Stephanie. 2015. Q-adjectives and the semantics of quantity. Journal of 
Structure preservation

Semantics 32(2). 221-273. doi:10.1093/jos/fft018.

Stateva, Penka. 2003. Superlative more. In Robert B. Young \& Yuping Zhou (eds.), Semantics and Linguistic Theory (SALT) XIII, 276-291. Cornell University, Ithaca, NY: CLC Publications. doi:10.3765/salt.v13i0.2893.

von Stechow, Arnim. 1984. Comparing semantic theories of comparison. Journal of Semantics 3(1). 1-77. doi:10.1093/jos/3.1-2.1.

Sutton, Peter R. \& Hana Filip. 2016. Countability: Individuation and context. In A. Foret, G. Morrill, R. Muskens, R. Osswald \& S. Pogodalla (eds.), Formal Grammar. fg 2016, fg 2015., vol. 9804 Lecture Notes in Computer Science, Berlin, Heidelberg: Springer.

Szabolcsi, Anna. 2012. Compositionality without word boundaries: (the) more and (the) most. In Semantics and Linguistic Theory (SALT) xx, 1-25. Cornell University, Ithaca, NY: CLC Publications. doi:10.3765/salt.v22i0.2629.

Wellwood, Alexis. 2012. Back to basics: more is always much-er. In E. Chemla, V. Homer \& G. Winterstein (eds.), Sinn und Bedeutung (SuB) 17, Paris: ENS.

Wellwood, Alexis. 2014. Measuring predicates. College Park, MD: University of Maryland dissertation. doi:10.13016/M2J30N.

Wellwood, Alexis. 2015. On the semantics of comparison across categories. Linguistics and Philosophy 38(1). 67-101. doi:10.1007/s10988-015-9165-0.

Wellwood, Alexis. forthcoming. The semantics of more. Studies in Semantics and Pragmatics. Oxford UK: Oxford University Press.

Wellwood, Alexis, Valentine Hacquard \& Roumyana Pancheva. 2012. Measuring and comparing individuals and events. Journal of Semantics 29(2). 207-228. doi:10.1093/jos/ffr006.

Winter, Yoad. 2001. Flexibility principles in Boolean semantics: The interpretation of coordination, plurality, and scope in natural language. Boston MA: MIT Press.

Alexis Wellwood

School of Philosophy

University of Southern California

3709 Trousdale Parkway

Los Angeles CA, 90089

wellwood@usc.edu 